\title{
Fragment Isospin as a Probe of Heavy-Ion Collisions
}

\author{
H. Xu, R. Alfaro, B. Davin, L. Beaulieu, Y. Larochelle, f T. Lefort, R. Yanez, and R.T. de Souza \\ Department of Chemistry and Indiana University Cyclotron Facility, \\ Indiana University, Bloomington, IN 47405
}

\author{
T.X. Liu, X.D. Liu, W.G. Lynch, R. Shomin, W.P. Tan, M.B. \\ Tsang, A. Vander Molen, A. Wagner, H.F. Xi, and C.K. Gelbke \\ National Superconducting Cyclotron Laboratory and Department of Physics and Astronomy, \\ Michigan State University, East Lansing, MI 48824 \\ R.J. Charity and L.G. Sobotka \\ Department of Chemistry, Washington University, St. Louis, MO 63130 \\ A.S. Botvina \\ Gesellschaft für Schwerionenforschung, 64291 Darmstadt, Germany and \\ Institute for Nuclear Research, Russian Academy of Science, 117312 Moscow, Russia
}

(Dated: October 22, 2018)

\begin{abstract}
Isotope ratios of fragments produced at mid-rapidity in peripheral and central collisions of ${ }^{114} \mathrm{Cd}$ ions with ${ }^{92} \mathrm{Mo}$ and ${ }^{98} \mathrm{Mo}$ target nuclei at $\mathrm{E} / \mathrm{A}=50 \mathrm{MeV}$ are compared. Neutron-rich isotopes are preferentially produced in central collisions as compared to peripheral collisions. The influence of the size (A), density, N/Z, $\mathrm{E}^{*} / \mathrm{A}$, and $\mathrm{E}_{\text {flow }} / \mathrm{A}$ of the emitting source on the measured isotope ratios was explored by comparison with a statistical model (SMM). The mid-rapidity region associated with peripheral collisions does not appear to be neutron-enriched relative to central collisions.
\end{abstract}

PACS numbers: 25.70.Mn

When projectile and target nuclei collide at intermediate energies $(20 \leq \mathrm{E} / \mathrm{A} \leq 100 \mathrm{MeV})$, a characteristic signature is the copious production of intermediate mass fragments (IMF:3 $\leq \mathrm{Z} \leq 20$ ). Continuum models of nuclear matter have recently been used to propose that nuclear matter at high excitation may fractionate[1], that is separate into liquid and gaseous phases with different $\mathrm{N} / \mathrm{Z}$. Driven by the density dependence of the asymmetry energy, such a process predicts formation of a neutron-rich gas and a proton-rich liquid[2]. For peripheral and midcentral collisions, where a low density zone between the projectile and target nuclei is formed, the density dependence of the asymmetry energy may also result in neutron enrichment of the mid-rapidity region. Dynamical stochastic transport models make specific predictions for neutron enrichment with increasing centrality [3, 4]. It is therefore interesting to examine the isotopic composition of fragments at mid-rapidity and determine the dependence of neutron enrichment on centrality. Although the elemental breakup of the mid-rapidity zone in peripheral collisions has been previously investigated [5], only recently has the $\mathrm{N} / \mathrm{Z}$ composition of $\mathrm{IMFs}$ in this region become available. While investigation of similar phenomena at lower incident energies [6, 7] suggests that frag-

\footnotetext{
* Present address: Universite Laval, Quebec, Canada.

${ }^{\dagger}$ Present address: Universite de Caen, Caen, France.

${ }^{\ddagger}$ Present address: Universidad de Chile, Santiago, Chile.

§Present address: Institute of Nuclear and Hadron Physics, Forschungszentrum, Rossendorf, Dresden, Germany.
}

ment emission might have a non-equilibrium character, it is instructive to first examine the experimental findings within a statistical context.

For peripheral and mid-central collisions of two heavyions at intermediate energies, it is well established that the nature of the collision is dissipative and largely binary 8, 9, 10]. Following the interaction phase in which the kinetic energy of relative motion is converted into intrinsic excitation (manifested by the velocity damping of the projectile) and pre-equilibrium emission, the excited projectile-like (PLF) and target-like (TLF) reaction partners decay by statistical emission of neutrons and light charged particles. When the IMF yield is examined, however, one observes a large excess over the sequential decay component located at mid-rapidity between the PLF and TLF 5, 7, 11, 12]. For light clusters $(\mathrm{Z}=1,2)$, neutron-rich clusters are particularly prevalent at mid-rapidity 13. Whether this neutron enrichment of light clusters signals an isospin fractionation of the system remains a topic of much debate.

In order to probe the $\mathrm{N} / \mathrm{Z}$ of the mid-rapidity region, we investigated IMF and light charged particle (LCP: $1 \leq \mathrm{Z} \leq 2$ ) emission in the reaction ${ }^{114} \mathrm{Cd}+{ }^{92} \mathrm{Mo}$ and ${ }^{114} \mathrm{Cd}+{ }^{98} \mathrm{Mo}$ at $\mathrm{E} / \mathrm{A}=50 \mathrm{MeV}$. In an exclusive $4 \pi$ setup we detected isotopically identified LCPs and IMFs with $\mathrm{Z} \leq 9$ in the angular range $7^{\circ} \leq \theta_{l a b} \leq 58^{\circ}$. These fragments were detected with the high resolution silicon-strip array LASSA (Si(IP)-Si(IP)-CsI(Tl)/PD) which had an energy threshold of 2 and $4 \mathrm{MeV} / \mathrm{u}$ for $\alpha$ and carbon fragments, respectively 15, 16. Forward-moving projectile-like fragments were identified in an annular 


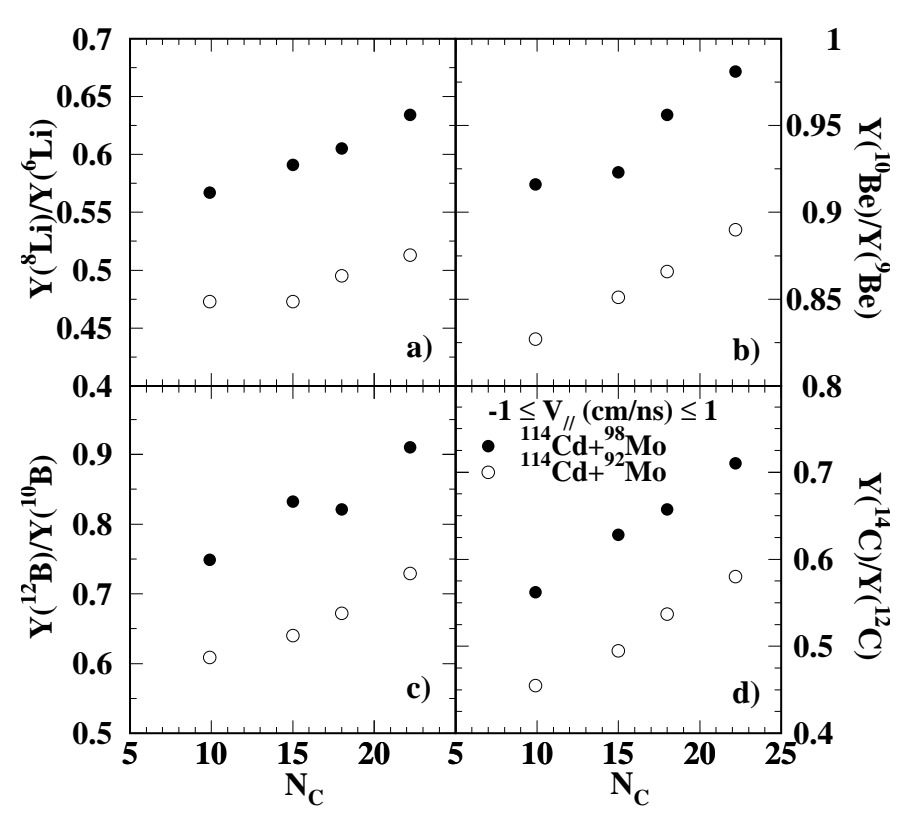

FIG. 1: Ratio of isotopic yields for fragments with $3 \leq \mathrm{Z} \leq 6$ at mid-rapidity $(-1 \leq \mathrm{v} / /(\mathrm{cm} / \mathrm{ns}) \leq 1)$ as a function of $\mathrm{N}_{c}$ for the reactions ${ }^{114} \mathrm{Cd}+{ }^{92} \mathrm{Mo}$ and ${ }^{114} \mathrm{Cd}+{ }^{98} \mathrm{Mo}$ at $\mathrm{E} / \mathrm{A}=50$ $\mathrm{MeV}$.

$\mathrm{Si}(\mathrm{IP}) / \mathrm{CsI}(\mathrm{Tl})$ ring detector $2.1^{\circ} \leq \theta_{\text {lab }} \leq 4.2^{\circ}$, which provided unit $\mathrm{Z}$ resolution for $\mathrm{Z} \leq 48$. Additionally, charged particles were measured in the range $5^{\circ} \leq \theta_{l a b} \leq 168^{\circ}$ by the Miniball/Miniwall array, allowing global event characterization 17.

We examined the isotopic composition of fragments at mid-rapidity by first selecting collisions on the basis of the charged particle multiplicity, $\mathrm{N}_{C}$. When $\mathrm{N}_{C} \leq 13$ ("peripheral collisions"; $\left\langle\mathrm{N}_{C}\right\rangle=9.9$ ), a fragment emission pattern is observed that is consistent with fragment emission following a dissipative, largely binary collision between the projectile and target nuclei. In contrast, when $\mathrm{N}_{C}>19$ ("central" collisions; $\left\langle\mathrm{N}_{C}\right\rangle=22.2$ ), the emission pattern for $\mathrm{He}$ and $\mathrm{Li}$ fragments is broad and featureless with substantial emission near the center-of-mass velocity. By relating the multiplicity distribution to a reduced impact parameter scale 18], we deduced that the multiplicity interval $\mathrm{N}_{C} \leq 13$ corresponds to $\left\langle\mathrm{b} / \mathrm{b}_{\max }\right\rangle=0.65$, while $\mathrm{N}_{C} \geq 20$ corresponds to $\left\langle\mathrm{b} / \mathrm{b}_{\max }\right\rangle=0.26$.

We further selected fragments by focusing on those detected in the interval $-1 \leq \mathrm{V}_{/ /}(\mathrm{cm} / \mathrm{ns}) \leq 1$, where $\mathrm{V}_{/ /}$is the longitudinal component of a fragment's velocity in the center-of-mass, and examined different isotope ratios as a function of $\mathrm{N}_{C}$. We subsequently utilize the nomenclature $\mathrm{HH}$ (heavy-heavy) and HL (heavy-light) to represent the ${ }^{114} \mathrm{Cd}+{ }^{98} \mathrm{Mo}$ and ${ }^{114} \mathrm{Cd}+{ }^{92} \mathrm{Mo}$ reactions, respectively. It should be noted that for the $\mathrm{HH}$ system, the $\mathrm{N} / \mathrm{Z}$ of the projectile and target is essentially the same $\left((\mathrm{N} / \mathrm{Z})_{\text {proj }}=1.375 ;(\mathrm{N} / \mathrm{Z})_{\text {targ }}=1.333\right)$ thus providing little driving force for neutron enrichment of mid-rapidity due to isospin equilibration.
The systematic isotopic behavior at mid-rapidity is shown in Fig. 1 where we explore the the dependence of mid-rapidity neutron enrichment on both centrality and the neutron content of the original system by examining isotope ratios for $3 \leq \mathrm{Z} \leq 6$. Solid symbols depict the dependence of the yield ratio $\mathrm{Y}\left({ }^{A 2} \mathrm{Z}\right) / \mathrm{Y}\left({ }^{A 1} \mathrm{Z}\right)$, where $\mathrm{A} 2>\mathrm{A} 1$, for the neutron-rich HH system while open symbols represent the same ratio for the relatively neutrondeficient HL system. In all cases shown in Fig. 1, a roughly linear increase of the relative yield for neutronrich fragments as a function of on $\mathrm{N}_{C}$ is observed. As large $\mathrm{N}_{C}$ can be associated with more central, higher excitation collisions, it appears that neutron-rich fragments at mid-rapidity are preferentially produced under these conditions. If the $\mathrm{N} / \mathrm{Z}$ at mid-rapidity for peripheral and central collisions is the same, and secondary decay contributions are comparable for the two cases, one would expect the relative probability for neutronrich fragments as compared to neutron-poor fragments to be constant. Should secondary decay increase in importance for more central collisions due to higher excitation, neutron-rich fragments would be favored in peripheral collisions as compared to central collisions. This observation that neutron-rich fragments are preferentially produced in central collisions might suggest that peripheral collisions are characterized by a lower $\mathrm{N} / \mathrm{Z}$ than central collisions. However, it is also conceiveable that higher excitation for peripheral collisions supresses the survival of neutron-rich fragments.

It is evident in Fig. 1 that while both the $\mathrm{HH}$ and HL systems manifest the same trend with $\mathrm{N}_{C}$, the relative yield of the neutron-rich species is enhanced in the $\mathrm{HH}$ system as compared to the HL system. Moreover, the enhancement observed for the $\mathrm{HH}$ system as compared to the HL system for large $\mathrm{N}_{C}$ that has been previously observed 2, 14] occurs with essentially the same magnitude even for the peripheral (low $\mathrm{N}_{C}$ ) case. This result indicates that the initial difference in $\mathrm{N} / \mathrm{Z}$ between the $\mathrm{HH}$ and HL systems influences the relative production of fragments at mid-rapidity, and persists from peripheral collisions to central collisions. For the following analysis we determined that the HH and HL systems provided essentially the same results. For simplicity, we therefore focus on the HH system.

To investigate the isotopic yields associated with peripheral and central collisions further, we compared our results with a statistical multifragmentation model (SMM) 19, 20. In this microcanonical model, the statistical partition into fragments can be calculated for a source characterized by a size of $(\mathrm{A})$, a density $\left(\rho / \rho_{0}\right)$, neutron-to-proton ratio $(\mathrm{N} / \mathrm{Z})$, with a given excitation energy $\left(\mathrm{E}^{*} / \mathrm{A}\right)$, and possibly a radial flow energy $\left(\mathrm{E}_{\text {flow }} / A\right)$. For the present analysis we have assumed that fragment production at mid-rapidity results from a single mid-rapidity source. This assumption of a single source, formed by the overlap of the projectile and target nuclei, is somewhat simplistic. Nevertheless, it allows us to consider the general factors governing fragment 


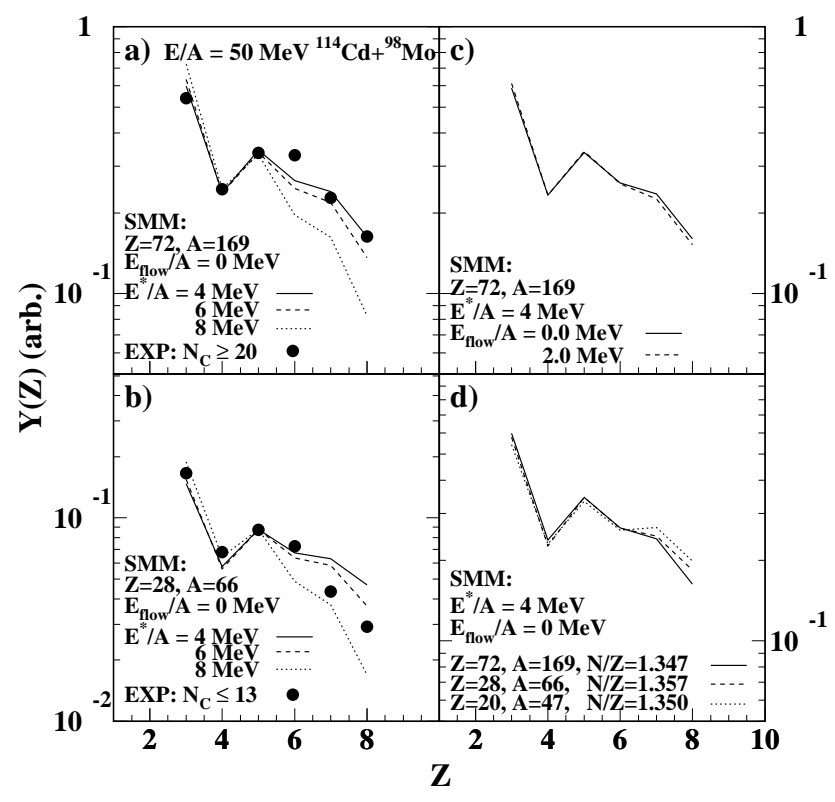

FIG. 2: Panel a: Comparison of the measured $\mathrm{Z}$ distribution associated with central collisions with the predicted $\mathrm{Z}$ distribution of the SMM model for a source with $\mathrm{Z}=72, \mathrm{~A}=169$ and $\mathrm{E}^{*} / \mathrm{A}=4,6$, and $8 \mathrm{MeV}$. Panel b: Comparison of the measured $\mathrm{Z}$ distribution associated with peripheral collisions with the predicted $\mathrm{Z}$ distribution of the SMM model for a source with $\mathrm{Z}=28, \mathrm{~A}=66$ and $\mathrm{E}^{*} / \mathrm{A}=4,6$, and $8 \mathrm{MeV}$. Panel c: Investigation of the influence of radial flow on the $\mathrm{Z}$ distributions predicted by the SMM model for a source with $\mathrm{Z}=72$, $\mathrm{A}=169, \mathrm{E}^{*}=4 \mathrm{MeV} . \mathrm{E}_{\text {flow }} / \mathrm{A}$ was selected to be 0 and 2 $\mathrm{MeV}$. Panel d: Investigation of the influence of source size with approximately constant $\mathrm{N} / \mathrm{Z}$ on the $\mathrm{Z}$ distributions as predicted by the SMM model.

production at mid-rapidity. It has recently been suggested that fragment production at mid-rapidity may be influenced, particularly for peripheral collisions, by the Coulomb proximity-induced emission of the projectilelike and target-like fragments 21. Detailed comparisons with such a scenario are beyond the scope of the present investigation but are being pursued and will be presented in a forthcoming publication. All model calculations presented have been filtered by the angular acceptance of LASSA $\left(7^{\circ} \leq \theta_{l a b} \leq 58^{\circ}\right)$ as well as the restriction that $1 \leq \mathrm{V}_{/ /}(\mathrm{cm} / \mathrm{ns}) \leq 1$.

In order to explore the sensitivity of the measured isotopic yields to the excitation $\left(\mathrm{E}^{*} / \mathrm{A}\right)$ and $\mathrm{N} / \mathrm{Z}$ of the emitting source, it was first necessary to constrain the size (A), density $\left(\rho / \rho_{0}\right)$, and radial flow energy $\mathrm{E}_{\text {flow }} / \mathrm{A}$ in the SMM model. We first compared the experimentally measured $\mathrm{Z}$ distributions in the range $-1 \leq \mathrm{V} / /(\mathrm{cm} / \mathrm{ns}) \leq 1$ with the predictions of the SMM model. The experimental Z distribution observed for central and peripheral reactions are represented as the solid symbols in Fig. 2. These data have been normalized by the number of events in each case. The experimental data exhibit an overall "exponential" decrease in yield with increasing Z.

In the SMM calculations, for the case of central collisions, we assumed the source size was $80 \%$ the size of the composite system with $\mathrm{N} / \mathrm{Z}$ equal to the composite system. For this case we display the $\mathrm{Z}$ distribution predicted by the SMM model for $\mathrm{E}^{*} / \mathrm{A}=4,6$, and $8 \mathrm{MeV}$ when $\rho / \rho_{0}=1 / 6$. The presented yields have been normalized to the yield for $\mathrm{Z}=5$ at mid-rapidity allowing comparison of the shape of the $\mathrm{Z}$ distribution. The calculations with $\mathrm{E}^{*} / \mathrm{A}=4-6 \mathrm{MeV}$ provide reasonable agreement with the experimental data (Fig. 2a) ,however, for $\mathrm{E}^{*} / \mathrm{A}=8$ $\mathrm{MeV}$ the predicted $\mathrm{Z}$ distribution is significantly steeper than the measured one. As expected, we observe that the slope of the $\mathrm{Z}$ distribution is mainly sensitive to the excitation of the source.

In order to estimate the size of the mid-rapidity source associated with peripheral collisions, we have examined the detected charge, $\mathrm{Z}_{\text {sum }}$, in the ring counter with the appropriate velocity and associated multiplicity $\mathrm{N}_{c}$. We then assumed that the $\mathrm{N} / \mathrm{Z}$ of this PLF was the same as the $\mathrm{N} / \mathrm{Z}$ of the projectile. By utilizing a participantspectator model we were able to estimate the contribution of neutrons and protons to mid-rapidity from the target nucleus. By this means, we estimated the size of the mid-rapidity source to be $A=66$.

For the case of peripheral collisions (Fig. 2b), with $\rho / \rho_{0}=1 / 6$ and a source size of $A=66, Z=28(\mathrm{~N} / \mathrm{Z}=1.357)$, we show the extent to which the $\mathrm{Z}$ distribution is modified as $\mathrm{E}^{*} / \mathrm{A}$ increases. For this source an excitation energy $\mathrm{E}^{*} / \mathrm{A} \sim 6-8 \mathrm{MeV}$ provides the best agreement with the experimental results.

We have also explored the sensitivity of the $\mathrm{Z}$ distribution to the source $\mathrm{N} / \mathrm{Z}$ and its size. While selection of a smaller $N / Z$ (neutron-poor) source does supress the yield for larger $\mathrm{Z}$ fragments, the overall modification of the $\mathrm{Z}$ distribution for the elements shown is not strong. The influence of radial flow, reported in similar reactions, on our results has also been examined. As shown in Fig. 2c, the $\mathrm{Z}$ distribution predicted by the SMM is essentially insensitive to this collective motion i.e. the fragment velocities are already well above the experimental acceptance. The influence of source size (assuming a near constant $\mathrm{N} / \mathrm{Z}$ ) is shown in Fig. 2d. For the range of source sizes assumed, which span the physically realistic range, the $\mathrm{Z}$ distribution is fairly independent of the source size with only a minor sensitivity for the largest fragments.

We also investigated whether the transverse velocity distributions, $\mathrm{V}_{\perp}$, for fragments emitted at mid-rapidity could be used to constrain the $\mathrm{E}^{*} / \mathrm{A}$ of the emitting source and determine if any radial flow is present for this reaction. Distributions for ${ }^{6} \mathrm{Li}$ and ${ }^{12} \mathrm{C}$, representative of other fragments, are shown in Fig. 3. In panel a), the experimental $\mathrm{V}_{\perp}$ distributions of ${ }^{6} \mathrm{Li}$ and ${ }^{12} \mathrm{C}$ fragments measured for central collisions $\left(\mathrm{N}_{C} \geq 20\right)$ are compared with the SMM calculations for the decay of the large source $(\mathrm{Z}=72, \mathrm{~A}=169)$ with $\mathrm{E}_{\text {flow }} / \mathrm{A}=0$ for $\mathrm{E}^{*} / \mathrm{A}=4,6$, and $8 \mathrm{MeV}$. Most clearly in the case of ${ }^{12} \mathrm{C}$, increased excitation energy results in a better reproduction of the 


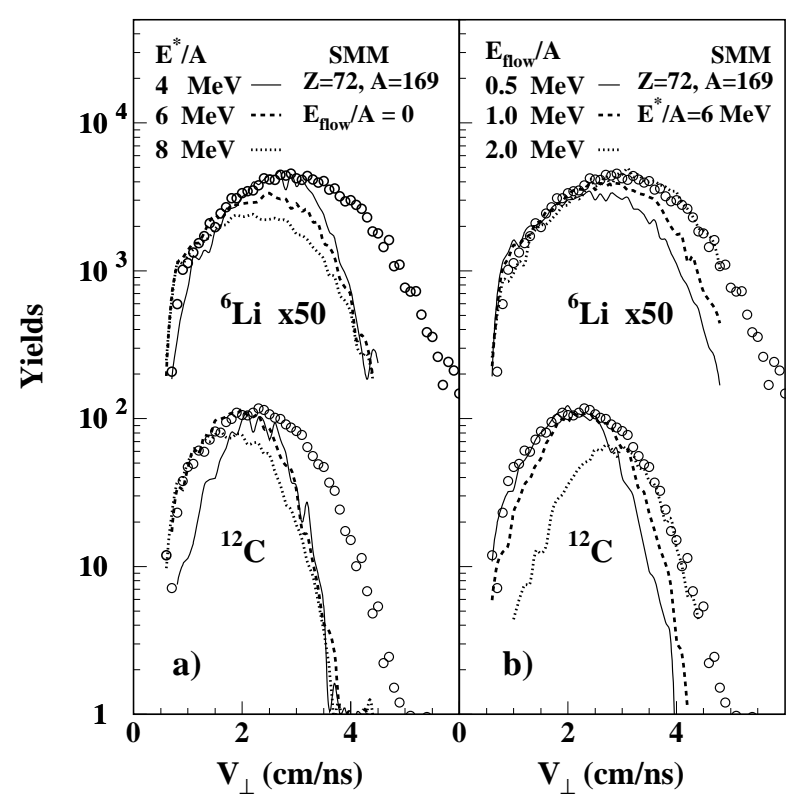

FIG. 3: Panel a: Comparison of $\mathrm{V}_{\perp}$ distributions associated with central collisions for ${ }^{6} \mathrm{Li}$ and ${ }^{12} \mathrm{C}$ fragments with the SMM predictions for a source $\left(\mathrm{Z}=72, \mathrm{~A}=169, \mathrm{E}_{\text {flow }} / \mathrm{A}=0\right.$ $\mathrm{MeV}$ ) with $\mathrm{E}^{*} / \mathrm{A}=4,6$, and $8 \mathrm{MeV}$. Panel b: Comparison of $\mathrm{V}_{\perp}$ distributions associated with central collisions for ${ }^{6} \mathrm{Li}$ and ${ }^{12} \mathrm{C}$ fragments with the SMM predictions for a source $\left(\mathrm{Z}=72, \mathrm{~A}=169, \mathrm{E}^{*} / \mathrm{A}=6 \mathrm{MeV}\right)$ with different amounts of radial flow energy, $\mathrm{E}^{*} / \mathrm{A}=0.5,1.0$, and $2 \mathrm{MeV}$.

low velocity portion of the $\mathrm{V}_{\perp}$ distributions. While $\mathrm{E}^{*} / \mathrm{A}$ $=4 \mathrm{MeV}$ appears to be too low an excitation to properly reproduce the low velocity portion of the spectrum, $\mathrm{E}^{*} / \mathrm{A}=6-8 \mathrm{MeV}$ provides an adequate description. In Fig. $3 \mathrm{~b}$, we demonstrate the influence of $\mathrm{E}_{\text {flow }} / \mathrm{A}$ on the $\mathrm{V}_{\perp}$ distribution. For the case of ${ }^{6} \mathrm{Li}$, increasing $\mathrm{E}_{\text {flow }} / \mathrm{A}$ from 0.5 to $2 \mathrm{MeV}$ provides a better description of the high velocity tail of the $\mathrm{V}_{\perp}$ distribution. The dramatic influence of $\mathrm{E}_{\text {flow }} / \mathrm{A}$ for heavy fragments is evident for ${ }^{12} \mathrm{C}$ where one observes that inclusion of a large flow for such fragments substantially shifts the $\mathrm{V}_{\perp}$ distribution to higher values of $\mathrm{V}_{\perp}$, therefore resulting in a significant underprediction on the low $\mathrm{V}_{\perp}$ side. The difficulty in describing the entire $\mathrm{V}_{\perp}$ distribution for several different elements with a single value for the radial expansion energy is hardly surprising considering that in reality we deal with an ensemble of sources. In reality, all quantities such as $\mathrm{E}_{\text {flow }} / A$ are probably distributions which may in fact depend on particle type. In this analysis we only attempt to describe the average behavior which affects the bulk of the cross-section. As indicated in Fig. 3, the bulk of the cross-section in the $\mathrm{V}_{\perp}$ distributions for ${ }^{6} \mathrm{Li}$ and ${ }^{12} \mathrm{C}$ are consistent with zero to modest flow energy i.e. $\mathrm{E}_{\text {flow }} / A=0-0.5 \mathrm{MeV}$.

Having constrained the source size, the excitation energy, and the flow energy for both the peripheral and central cases at mid-rapidity, we examined the sensitivity

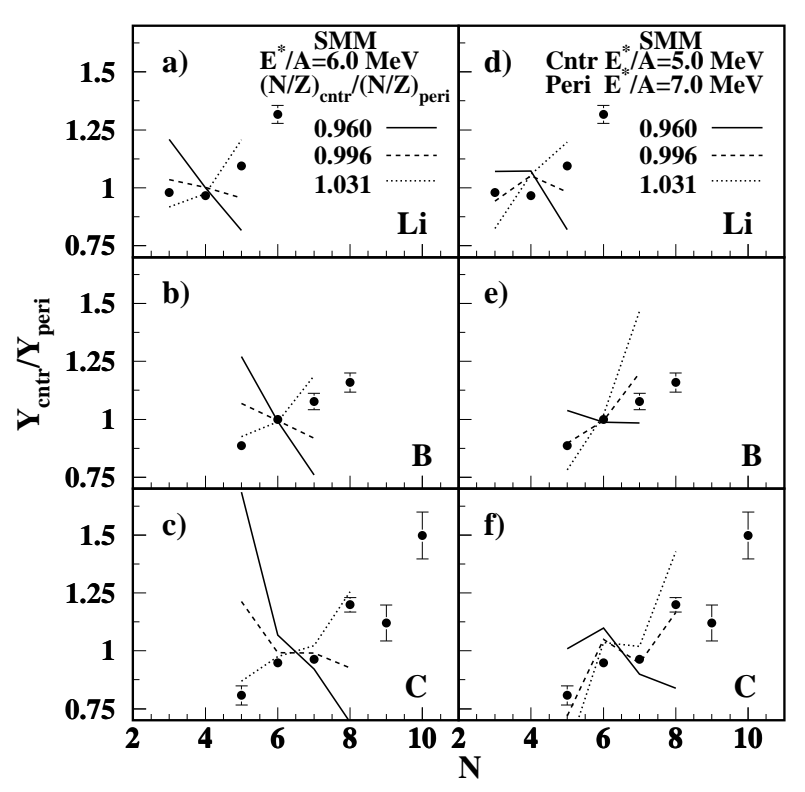

FIG. 4: Relative yield at mid-rapidity for isotopes of Li, B, and $\mathrm{C}$ associated with central and peripheral collisions. Panels a-c): SMM calculations assume both peripheral and central sources have $\mathrm{E}^{*} / \mathrm{A}=6 \mathrm{MeV}$ and $\mathrm{E}_{\text {flow }} / \mathrm{A}=0$. Panels d-f): The central and peripheral sources are assumed to have $\mathrm{E}^{*} / \mathrm{A}=5$ and $7 \mathrm{MeV}$, respectively, and $\mathrm{E}_{\text {flow }} / \mathrm{A}=0$. Solid, dashed, and dotted lines represent varying degrees of neutron enrichment of the peripheral source as compared to the central source.

of the ratio $\mathrm{Y}_{c n t r} / \mathrm{Y}_{\text {peri }}$ as a function of fragment neutron number $\mathrm{N}$, for fragments with $\mathrm{Z}=3-6$. The experimental results and the corresponding SMM calculations are presented in Fig. 4. The measured and predicted yields of each element for both peripheral and central collisions have been individually normalized, thus providing a comparison of isotopic predictions that is insensitive to the prediction of elemental yields. One observes that in all cases, the quantity $\mathrm{Y}_{c n t r} / \mathrm{Y}_{\text {peri }}$ for the experimental data manifests a general increase with increasing neutron number.

Following our constraint of the $\mathrm{E}^{*} / \mathrm{A}$ and $\mathrm{E}_{\text {flow }} / \mathrm{A}$ as described above, we have chosen two different frameworks within which to examine our results. In the first case, depicted in Fig. 4a-c, we assumed that the $\mathrm{E}^{*} / \mathrm{A}$ of the mid-rapidity region for both peripheral and central collisions is the same, i.e. $\mathrm{E}^{*} / \mathrm{A}=6.0 \mathrm{MeV}$, with a zero radial flow. The $\mathrm{Z}$ and $\mathrm{A}$ of the central source was fixed at $Z=72$ and $A=169$. For the peripheral source, the size of the source was taken to be $A=66$ while the $\mathrm{Z}$ was varied between $\mathrm{Z}=27-29$. The solid, dashed, and dotted lines indicate the results when $(\mathrm{N} / \mathrm{Z})_{\text {cntr }} /(\mathrm{N} / \mathrm{Z})_{\text {peri }}$ $=0.960,0.996$, and 1.031 , respectively, i.e. the peripheral source changes from being neutron-rich to being neutrondeficient as compared to the central source. The overall 
behavior of the SMM calculations can be summarized as follows: The slope of the quantity $\mathrm{Y}_{c n t r} / \mathrm{Y}_{\text {peri }}$ as a function of neutron number depends on the ratio of the $\mathrm{N} / \mathrm{Z}$ of the central and peripheral sources. As one goes from a neutron-rich peripheral source to a neutron-deficient peripheral source (with a constant $\mathrm{N} / \mathrm{Z}$ central source), the slope changes from negative to positive. The experimental data indicates that within this framework the peripheral source is slightly neutron-deficient as compared to the central source.

In Fig. 4d-f we present a second scenario in which we allow the $\mathrm{E}^{*} / \mathrm{A}$ of the peripheral and central cases to be different. For this scenario, the $\mathrm{E}^{*} / \mathrm{A}$ was determined by comparing the predicted $\mathrm{Z}$ distributions to the experimental data. As a result of these comparisons, we deduced $\mathrm{E}^{*} / \mathrm{A}=5.0 \mathrm{MeV}$ for the central case and $\mathrm{E}^{*} / \mathrm{A}$ $=7.0 \mathrm{MeV}$ for the peripheral case. Under the assumption of these two different temperatures, comparison with the SMM calculations indicates that overall the data is compatible only with the case of no significant neutron enrichment of the peripheral source in comparison to the central source. In both scenarios presented we have determined that our conclusions are independent of our assumptions of the breakup density, source size, and radial flow.

Within a strictly geometrical picture of the collision the $\mathrm{N} / \mathrm{Z}$ of the system remains constant in the overlap region. As indicated by the comparison of the measured $\mathrm{Z}$ distributions with the SMM calculations, the initial excitation of both the peripheral and central sources may well be comparable. Secondary decay effects are thus not expected to dominate the behavior of the ratio $\mathrm{Y}_{\text {central }} / \mathrm{Y}_{\text {peripheral }}$ with neutron number. Rather, the trend of this ratio with neutron number may suggest that the mid-rapidity source that produces fragments in peripheral collisions is neutron-deficient as compared to the mid-rapidity source for central collisions. Alternatively, the mid-rapidity source in peripheral and central collisions may have the same N/Z. In this case, however, the $\mathrm{E}^{*} / \mathrm{A}$ of the peripheral source must exceed the $\mathrm{E}^{*} / \mathrm{A}$ of the central source.

While at the present time neither possibility can be eliminated, it is interesting to speculate on the possible reason the mid-rapidity region may be neutron-deficient for peripheral collisions as compared to central collisions as evinced by fragment isotope yields. One scenario consistent with these results is that the initial source at midrapidity for both the peripheral and central cases is consistent with the $\mathrm{N} / \mathrm{Z}$ of the system. Prior to fragment formation, however, neutrons are preferentially depleted from this system thus affecting the $\mathrm{N} / \mathrm{Z}$ ratio. As shown in Fig. 4, removal of a single neutron has a measurable impact on the observed isotopic yields.

In summary, we have probed the $\mathrm{N} / \mathrm{Z}$ composition at mid-rapidity for both peripheral and central collisions by examining the $\mathrm{N} / \mathrm{Z}$ ratio of fragments with $3 \leq \mathrm{Z} \leq 6$. Neutron-rich fragments are preferentially produced for central collisions as opposed to peripheral collisions. This supression of neutron-rich fragments in peripheral collisions can be related to either a neutron-deficient source at mid-rapidity for peripheral collisions as compared to central collisions or a more excited mid-rapidity region in peripheral collisions.

We would like to acknowledge the valuable assistance of the K1200 cyclotron staff at MSU-NSCL for providing the high quality beams that made this experiment possible. We are grateful to Dr. Jan Toke for his help with the data acquisition. One of the authors ( $R d S)$ is especially grateful to Commissariat a l' Energie Atomique and G.A.N.I.L. (France) for support enabling this work during a sabbatical leave. A.S.B. thanks Indiana University for warm hospitality and support during his visit. This work was supported by the U.S. Department of Energy under DE-FG02-92ER40714 (IU), DE-FG0287ER-40316 (WU) and the National Science Foundation under Grant No. PHY-95-28844 (MSU).
[1] H. Müller and B. D. Serot, Phys. Rev. C,52, 2072 (1995).

[2] H.Xu et al., Phys. Rev. Lett., 85, 716 (2000).

[3] L.G. Sobotka, Phys. Rev. C 50, R1272 (1994).

[4] M. di Toro et al., Nucl. Phys. A681, 426c (2000).

[5] C. P. Montoya et al., Phys. Rev. Lett., 73, 3070 (1994).

[6] G. Casini et al., Phys. Rev. Lett. 71, 2567 (1993).

[7] J. Toke et al., Phys. Rev. Lett. 75, 2920 (1995).

[8] B. Lott et al., Phys. Rev. Lett. 68, 3141 (1992).

[9] L. Beaulieu et al., Phys. Rev. Lett. 77, 462 (1996).

[10] E. Plagnol et al., Phys. Rev. C 61, 014606 (2000).

[11] J. Lukasik et al., Phys. Rev. C 55, 1906 (1997).

[12] E. Plagnol et al., Phys. Rev. C 61, 014606 (1999).

[13] J. F. Dempsey et al., Phys. Rev. C. 54, 1710 (1996).
[14] G. J. Kunde et al., Phys. Rev. Lett. 77, 2897 (1996).

[15] B. P. Davin, et al., Nucl. Instr. and Meth. A*** (2001) in press.

[16] A. Wagner, et al., Nucl. Instr. and Meth. A456, 290 (2001).

[17] R. T. de Souza et al., Nucl. Instr. Meth. A295, 109 (1990).

[18] C. Cavata et al., Phys. Rev. C 42, 1760 (1990).

[19] J. P. Bondorf et al., Phys. Rep. 257, 133 (1995).

[20] A. S. Botvina and I. N. Mishustin, Phys. Rev. C 63, 061601(R) (2001).

[21] A. S. Botvina et al., Phys. Rev. C 59, 3444 (1999). 